\title{
Facial asymmetry in head and neck rhabdomyosarcoma survivors
}

Reineke A. Schoot MD PhD ${ }^{1}$, Marinka Hol MD², Johannes M. Merks MD PhD ${ }^{1}$, Michael Suttie $\mathrm{MSc}^{3}$, Olga Slater $\mathrm{MD}^{4}$, Marinde van Lennep $\mathrm{MD}^{1}$, Saskia M.J. Hopman MD PhD ${ }^{5}$, Jonathan Syme-Grant $\mathrm{MD}^{6}$, Ludi E. Smeele MD PhD ${ }^{2}$, Koos H. Zwinderman $\mathrm{PhD}^{7}$, Huib N. Caron MD $\mathrm{PhD}^{1}$, Peter Hammond $\mathrm{PhD}^{3}$

1 Department of Paediatric Oncology, Emma Children's Hospital, Academic Medical Centre, Amsterdam, The Netherlands

2 Department of Head and Neck Surgery, Antoni van Leeuwenhoek The Netherlands Cancer Institute- Academic Medical Centre, Amsterdam, The Netherlands

3 Nuffield Department of Obstetrics and Gynaecology, University of Oxford, Oxford, UK

4 Department of Paediatric Oncology, Great Ormond Street Hospital for Children NHS Foundation Trust, London, United Kingdom

5 PLEASE PROVIDE INSTITUTION

6 Craniofacial Unit, Great Ormond Street Children's Hospital for Children NHS Foundation Trust, London, United Kingdom

7 Department of Clinical Epidemiology and Bio-Statistics, Academic Medical Centre, Amsterdam, The Netherlands

\section{Acknowledgements}

Natalia Katting, Freida Angullia

\section{Corresponding author:}

Professor Peter Hammond

Nuffield Department of Obstetrics and Gynaecology

University of Oxford

Women's Centre, John Radcliffe Hospital

Oxford OX3 7DQ, United Kingdom

Email: peter.hammond@obs-gyn.ox.ac.uk Telephone: xx Fax: xx

Running head Facial asymmetry in head and neck rhabdomyosarcoma survivors Conflict of interest: None 


\section{Abstract (252 words)}

\section{PURPOSE}

Radiotherapy is essential for achieving and maintaining local control in head and neck rhabdomyosarcoma patients. However, radiotherapy may cause outgrowth disturbances of facial bone and soft tissue, resulting in facial asymmetry. The aim of this study was to develop a method to visualise and measure facial asymmetry in head and neck rhabdomyosarcoma survivors using three dimensional (3D) imaging techniques.

\section{PATIENTS AND METHODS}

Facial deformity was evaluated in a multi-disciplinary clinical assessment of 75 head and neck rhabdomyosarcoma survivors, treated with external beam radiotherapy (EBRT, $n=26$ ) or Ablative surgery, MOulage brachytherapy and REconstruction (AMORE, $n=49$ ). Individual facial asymmetry was measured using 3D photogrammetry and expressed in a raw asymmetry index and a normalised sex-age-ethnicity matched asymmetry signature weight. Facial asymmetry was also compared between British and Dutch controls and between survivors and their matched controls.

\section{RESULTS}

Facial asymmetry was more pronounced with increasing age $(p<0.01)$ in British controls compared to Dutch controls $(p=0.04)$. Survivors developed more facial asymmetry than matched controls $(p<0.001)$. The clinical assessment of facial deformity correlated with the raw asymmetry index $(p<0.001)$. Survivors treated with EBRT displayed more facial deformity than survivors treated with AMORE $(p=0.039)$.

\section{CONCLUSION}

3D imaging can be used for objective measurement of facial asymmetry in head and neck RMS survivors. The raw asymmetry index correlated with a clinical assessment of facial deformity. Comparisons between treatment groups seemed inappropriate given the differences in facial asymmetry between British and Dutch controls. In future studies pretreatment images could act as matched controls for post-treatment evaluation. 


\section{INTRODUCTION (2569 words)}

Survival in head and neck rhabdomyosarcoma (RMS) patients is good, with overall survival rates around $79-97 \%$ (1-3). However, the majority of patients still need radiotherapy to achieve and maintain local control. Radiotherapy damages all tissues within the radiation field, including healthy tissues surrounding the tumour. Head and neck RMS patients are often young at diagnosis, with a median age of 5.5 years (1). At this age, radiotherapy can cause severe damage to the craniofacial bones resulting in asymmetrical growth of the face, often further exacerbated by hypoplasia of soft tissues.

In 1990, the AMORE treatment (Ablative surgery, MOulage brachytherapy and REconstruction) was developed, a local treatment equally effective compared to the international standard: external beam radiotherapy (EBRT) (3). Furthermore, moulage brachytherapy enables a focussed dose delivery with relative sparing of the healthy tissues causing fewer adverse events compared to EBRT (3). However, prior to this study no objective comparison of treatment induced facial asymmetry or hypoplasia had been performed.

Thus far, several (predominantly) retrospective studies have mentioned facial asymmetry or hypoplasia as a result of radiotherapy in head and neck RMS survivors (4-8). The detection of facial asymmetry or hypoplasia in these studies was also purely subjective and highly susceptible to inter-observer variability, with no definitions, absolute measurements nor grading scales being provided.

The aims of this study were as follows:

(i) to develop methodologies for visualising and quantifying facial asymmetry in head and neck RMS survivors and to compare asymmetry with age, sex and ethnicity matched healthy controls;

(ii) to compare measured asymmetry with a subjective clinical assessment of asymmetry;

(iii) to use the methodologies developed to compare asymmetry in survivors treated with EBRT (EBRT-based treatment) to those treated in a centre with the possibility of AMORE (AMORE-based treatment).

\section{PATIENTS AND METHODS}

Setting 
In a recent study published by our group, adverse events of local treatment were evaluated in a multi-disciplinary clinic comparing survival and adverse events between survivors treated with EBRT and compared to survivors treated in a centre with the availability of AMORE (AMORE-based treatment) (3). As part of this clinic, patients were invited for a 3D photograph to objectify further clinical observation of facial differences in this specific population.

\section{Survivors}

Patient recruitment was based at Great Ormond Street Hospital (GOSH) London and the Emma Children's Hospital-Academic Medical Centre (EKZ-AMC) Amsterdam. All children up to 18 years of age at diagnosis, presenting between January 1990 and December 2010 with newly diagnosed head and neck RMS (HNRMS) and at least two years after the end of treatment were eligible. As the AMC is a centre of expertise for orbital tumours, the EKZAMC cohort contained relatively more survivors of orbital RMS. Therefore, survivors of orbital RMS treated at Royal Marsden Hospital (RMH) Sutton, in the same time period, were also invited to visit the GOSH multi-disciplinary clinic. Patients with recurrent disease following prior radiotherapy, foreign patients referred solely for local treatment and patients living abroad at time of assessment were excluded. Written informed consent was obtained from all survivors (age $\geq 12$ ) and/or their guardians.

\section{Controls}

The control group consisted of 693 subjects: 283 were of Dutch descent and 410 of British descent. Subjects were recruited in the hospital and concerned medical staff or employees from one of the research departments. The remainder were unaffected parents and siblings of children with a genetic syndrome, attending an outpatient visit or a syndrome support group meeting. For the asymmetry signature analysis we included 446 controls: controls below four years and over 36 years were excluded because of insufficient controls at the extreme age values for defining age-sex-ethnicity matched means.

\section{RMS treatment}

Patients were staged and treated according to subsequent SIOP-MMT-89, -95, -98 (International Society of Paediatric Oncology-Malignant Mesenchymal Tumour group), and EpSSG-RMS-2005 (European paediatric Soft tissue sarcoma Study Group) protocols (9-11). 
After induction chemotherapy (two or three courses) patients were evaluated for local treatment; SIOP-MMT and EpSSG protocols withhold radiotherapy in patients with favourable prognostic characteristics in complete remission at this time point. When indicated, local treatment for GOSH/RMH patients included EBRT (EBRT-based treatment); patients treated at the EKZ-AMC received AMORE if feasible (AMORE-based treatment), that is, if a macroscopic radical resection and mould placement with adequate radiotherapy fields seemed possible based on imaging studies. If not, EKZ-AMC patients received EBRT or (in later years) proton beam treatment.

\section{Clinical assessment}

In this cross sectional study, adverse events (AE) of local RMS treatment were evaluated in a specialised head-and-neck multi-disciplinary clinic and graded according to the Common Terminology Criteria for Adverse Events (CTCAEv4.0, available at http://evs.nci.nih.gov/ftp1/CTCAE/About.html). Facial asymmetry and hypoplasia were scored as one item 'facial deformity':

Grade 1: cosmetically and functionally insignificant hypoplasia

Grade 2: deformity, hypoplasia, or asymmetry able to be covered

Grade 3: significant deformity, hypoplasia, or asymmetry, unable to be remediated by prosthesis or covered by clothing; disabling

Grade 4: orbital exenteration

Orbital exenteration results in asymmetry which cannot be covered and in blindness of at least one eye, and as there was no associated grading, we scored it as grade 4.

3D image capture and preparation

We captured 3D face images using commercial stereo-photogrammetric cameras (Canfield Imaging Systems, New Jersey, USA; 3dMD, Atlanta, USA). These devices typically capture more than 25,000 surface points on an adult face. One researcher (PH) manually annotated all surfaces with 22 landmarks, adopted because of their known reproducibility (Appendix 2). Subsequently, using the sparse set of landmarks, face surfaces were closely aligned using the Procrustes algorithm to induce a dense correspondence of tens of thousands of points across all images and an average face was then computed. Differences between the densely corresponded points on each surface and on the overall average face 
were subject to a principal component analysis (PCA) and we constructed a 3D dense surface model consisting of the minimal number of PCA modes accounting for more than $99 \%$ of shape variation. Each face surface can be resynthesized as a linear sum of so-called PCA related eigenfaces weighted by the associated PCA scores. More details are available elsewhere $(12,13)$.

\section{Visualisation and quantification of facial asymmetry}

We studied facial asymmetry by comparing original and reflected (mirrored) face surfaces: all left-right-paired landmarks were swapped, enabling a dense surface model to be generated for all original and all reflected face surfaces together. Euclidean distance, square root of sum of squared PCA score differences, between the DSM-based representations of the surface of a face and its mirrored form (represented as vectors of PCA scores) was used as an estimate of directional facial asymmetry (the raw asymmetry index) (14).

The vector difference between the DSM representation of a face and its mirrored form induces a deformation of the surface of the overall mean face. This deformation can be normalised locally at each densely corresponded point of the DSM with respect to similar localised deformations of the face surfaces of a set of age-sex-ethnicity matched controls. A visualisation, referred to here as a facial asymmetry signature, is produced by colour coding the localised difference using an appropriate scale, typically -2 SD to +2 SD, using red-blue to indicate difference at or beyond the extremes of the scale. We can also calculate an asymmetry signature weight as the square root of the summed squares of the localised differences. The earlier raw asymmetry index provides an overall estimate of the asymmetry of the face surface whereas the asymmetry signature weight takes into account the statistical significance of localised asymmetry.

\section{Statistical analyses}

Differences between EBRT-based and AMORE-based treatment groups, with respect to patient and treatment characteristics and distribution of $A E^{\prime} s$, were analysed with the Chi square test, Fisher's exact test or Mann-Whitney $U$ test, depending on the type of variable.

We evaluated the effect of age on asymmetry in British and Dutch controls separately, calculating the Spearman coefficient. We compared the difference in asymmetry index between the British and Dutch control population in a linear regression analysis and 
adjusted for age. For the comparison of the asymmetry index between survivors with EBRTbased treatment (British survivors) and AMORE-based treatment (Dutch survivors) we corrected for the effect of age and ethnicity on the asymmetry index. We compared the clinical asymmetry assessment between treatment groups with the Fisher's exact test.

\section{RESULTS}

\section{Patients}

Eighty survivors attended the head-and-neck specialised multi-disciplinary clinic and 75 (94\%) survivors had a 3D image captured (26 EBRT-based treatment, 49 AMORE-based treatment) (Table 1). Due to organisational issues, 5/31 (16\%) survivors with EBRT-based treatment did not have their image captured. Nineteen survivors were of non-European descent. Significantly more survivors with EBRT-based treatment were of non-European descent ( $42 \%$ and $16 \%$ respectively, $p=0.02)$. In the AMORE-based treatment group ten survivors were treated with radiotherapy more than once compared with none of the survivors in the EBRT-based treatment group $(p=0.02)$.

\section{Asymmetry}

\section{Control population}

We evaluated images of subjects with asymmetry indices outside the $95 \%$ confidence interval $(\mathrm{Cl})$ : all images were of good quality and represented true asymmetry (Appendix 1 ). The asymmetry index increased with age $(p<0.01)$ and was significantly higher in the British control population compared with the Dutch control population $(p=0.04)$. These results suggest survivors with a non-European background may need an ethnically matched control population (15). Therefore the 19 survivors from non-European descent (EBRT-based; N=11, AMORE-based; $\mathrm{N}=8$ ) were excluded from quantitative analyses.

\section{Facial asymmetry in survivors of head and neck RMS}

We plotted the raw asymmetry index for head and neck RMS survivors against age and corrected for the increase of facial asymmetry with increasing age (Figure 1). Furthermore, we described the characteristics for survivors with an asymmetry index $>5$ (Table 2).

The normalised asymmetry (for survivors of European background), matched for age, sex, and ethnicity, is visualised in Figure 2. Signature weight is presented in Figure 3. 
Signature weight for British survivors compared to British controls was significantly higher $(p<0.001)$ as was the signature weight for Dutch survivors compared to Dutch controls $(<0.001)$.

\section{Clinical assessment of asymmetry}

During a clinical assessment for adverse events of local treatment in a specialised head-and-neck clinic 72/75 (96\%) of survivors were evaluated for facial asymmetry or hypoplasia (scored as facial deformity). Median grade for the entire cohort of survivors was 1 (interquartile range (IQR) 0-3), median grade for survivors with EBRT-based treatment was 1.5 (IQR 0-3) and for survivors with AMORE-based treatment 1 (IQR 0-2). The severity of clinically detected asymmetry was significantly lower in the AMORE-based treatment group compared to the EBRT-based treatment group ( $p=0.039$ ).

We found a significant correlation between the raw asymmetry index and the clinical assessment of facial deformity, graded according to the CTC AE $(p<0.001$, Figure 4).

Comparison of asymmetry index and signature weight between survivors with EBRT-based and AMORE-based treatment

The average raw asymmetry index for controls, corrected for increasing asymmetry with age, was $-0.28(95 \% \mathrm{Cl}(-3.3,2.7))$ and for survivors in the EBRT-based treatment group $3.4(95 \% \mathrm{Cl}(-2.8,9.5))$ and the AMORE-based treatment group $3.6(95 \% \mathrm{Cl}(-6.6,13.8))$ respectively.

Due to the significant differences in asymmetry between British and Dutch controls, the heterogeneity in this cohort of survivors and the small number of survivors after exclusion of survivors of non-European descent we considered further comparisons not justified and confined analysis to a description of survivors with high (adjusted) asymmetry indices (Table 2).

\section{DISCUSSION}

Facial asymmetry can be visualised and quantified with 3D imaging techniques. We found that with increasing age, faces become more asymmetrical and asymmetry was stronger in British controls compared to Dutch controls. Survivors of head and neck RMS developed more pronounced facial asymmetry following local treatment compared to age, 
sex and ethnicity matched controls. Furthermore, we found a significant correlation in head and neck RMS survivors between the raw asymmetry index and the clinical assessment of facial deformity.

The majority of people exhibit facial asymmetry, some more than others. Therefore comparison to matched controls is essential for facial asymmetry assessment. Due to the statistically significant difference in facial asymmetry between British and Dutch controls, no direct comparison was possible between survivors treated in the EBRT-based (UK) and AMORE-based (The Netherlands) treatment group. When finding a difference in facial asymmetry between treatment groups, this may in fact be caused by inter-population differences. Multivariate analyses, correcting for inter-population differences, seemed not appropriate considering the small number of survivors and the heterogeneity in the cohorts under investigation. It is not surprising to find differences in facial appearance between populations of different ethnical backgrounds and previous studies have shown that these differences can be measured using 3D imaging techniques [15-17]. However, both controls from the UK and from The Netherlands are from the European subpopulation and live in close geometrical proximity. This finding may imply that even subpopulations from different regions, in the same country, may display facial differences. This further impedes 3D comparisons between different survivor populations, as differences in local treatment are often related to a centre of expertise, and thus to a certain geographical region. Furthermore, $25 \%$ of the survivors in this study were of non-European (or mixed) background, lacking a proper control group for comparison of facial asymmetry. Further studies are needed to investigate if larger control sets, including balanced (sub) populations of different and mixed backgrounds, may overcome these inter-population differences.

The colour marked images in Figure 3 give a good impression of the asymmetry and type of facial differences for the individual survivors in this cohort. Ideally, newly diagnosed patients would have their 3D images taken regularly: before and during treatment and at follow-up. The pre-treatment image could function as a matched control and colour mapping can be used for detection of facial outgrowth disturbances. These pre- and posttreatment images can be related to radiotherapy treatment plans and incorporated into a prediction model for radiotherapy induced outgrowth disturbances. This model could be used when comparing different local treatment modalities or for the design of radiotherapy treatment plans: reducing radiotherapy, provided that it is oncologically safe, on areas prone to growth disturbances. 
All survivors were scored for facial deformity, according to the CTCAE criteria, by a multidisciplinary team. We found a statistically significant correlation between the clinical assessment and the raw asymmetry index described in this study. At clinical assessment, facial deformity was more pronounced in survivors treated with EBRT-based treatment compared to survivors treated in the AMORE-based treatment group. Although we did not compare facial deformity by clinical assessment between British and Dutch controls, hypothetically, this difference in facial deformity may have been influenced by the stronger facial asymmetry in the British controls.

Another limitation of this study may be the assessment method for facial asymmetry. In this study we used a sparse set of manually placed landmarks to induce a dense correspondence of tens of thousands of face surface points. An alternative method has been suggested by Claes et al. where the dense correspondence of face surface points is induced by a semi-automated technique [18]. Although both techniques use dense correspondence, the semi-automated method may be less accurate in this specific population, as this technique loses its accuracy in strongly dysmorphic faces [19]. As is shown in Figure 3 several survivors display severe facial differences, such as an exenterated orbit, which may not be recognised by semi-automated techniques.

This study shows that 3D imaging can be used for objective measurement of facial asymmetry in head and neck RMS survivors. The raw asymmetry index correlated with clinical assessment of facial deformity. Comparisons between treatment groups were hampered by differences in facial asymmetry between British and Dutch controls. 3D imaging can be used in future studies for prediction models of radiotherapy treatment related outgrowth disturbances and personalised local treatment plans. 


\section{REFERENCES}

1. Oberlin O, Rey A, Sanchez de TJ, et al.: Randomized comparison of intensified six-drug versus standard three-drug chemotherapy for high-risk nonmetastatic rhabdomyosarcoma and other chemotherapy-sensitive childhood soft tissue sarcomas: long-term results from the International Society of Pediatric Oncology MMT95 study. J Clin Oncol 30:2457-2465, 2012

2. Raney RB, Walterhouse DO, Meza JL, et al.: Results of the Intergroup Rhabdomyosarcoma Study Group D9602 protocol, using vincristine and dactinomycin with or without cyclophosphamide and radiation therapy, for newly diagnosed patients with low-risk embryonal rhabdomyosarcoma: a report from the Soft Tissue Sarcoma Committee of the Children's Oncology Group. J Clin Oncol 29:1312-1318, 2011

3. Schoot RA, Slater O, Ronckers CM, et al.: Adverse events in long-term head and neck rhabdomyosarcoma survivors after external beam radiotherapy or AMORE treament. Eur J Cancer 51:1424-34

4. Childs SK, Kozak KR, Friedmann AM, et al.: Proton radiotherapy for parameningeal rhabdomyosarcoma: clinical outcomes and late effects. Int J Radiat Oncol Biol Phys 82:635-642, 2012

5. Estilo CL, Huryn JM, Kraus DH, et al.: Effects of therapy on dentofacial development in long-term survivors of head and neck rhabdomyosarcoma: the memorial sloan-kettering cancer center experience. J Pediatr Hematol Oncol $25: 215-222,2003$

6. Fiorillo A, Migliorati R, Vassallo $\mathrm{P}$, et al.: Radiation late effects in children treated for orbital rhabdomyosarcoma. Radiother Oncol 53:143-148, 1999

7. Fromm M, Littman P, Raney RB, et al.: Late effects after treatment of twenty children with soft tissue sarcomas of the head and neck. Experience at a single institution with a review of the literature. Cancer 57:2070-2076, 1986

8. Raney RB, Asmar L, Vassilopoulou-Sellin R, et al.: Late complications of therapy in 213 children with localized, nonorbital soft-tissue sarcoma of the head and neck: A descriptive report from the Intergroup Rhabdomyosarcoma Studies (IRS)-II and - III. IRS Group of the Children's Cancer Group and the Pediatric Oncology Group. Med Pediatr Oncol 33:362-371, 1999

9. Bisogno $G$, Bergeron $C$, Jenney $M$ : European paediatric soft tissue sarcoma study group RMS 2005: a protocol for non-metastatic rhabdomyosarcoma (Assessed 21 March 2016 at: https://www.clinicaltrialsregister.eu/ctrsearch/search?query=European+paediatric+soft+tissue+sarcoma+study+group+ RMS+2005) 
10. McDowell HP, Foot ABM, Ellershaw C, et al.: Outcomes in paediatric metastatic rhabdomyosarcoma: Results of The International Society of Paediatric Oncology (SIOP) study MMT-98. European Journal of Cancer 46:1588-1595, 2010

11. Stevens M, Rey A, Bouvet N, et al.: SIOP MMT 95: Intensified (6 drug) versus standard (IVA) chemotherapy for high risk non metastatic rhabdomyosarcoma (RMS). Journal of Clinical Oncology 22:8515, 2004

12. Hutton TJ, Buxton BF, Hammond P. Automated Registration of 3D Faces using Dense Surface Models. BMVC 1-10, 2003

13. Hammond $P$, Suttie $M$ : Large-scale objective phenotyping of 3D facial morphology. Hum Mut 33 (5):817-825, 2012

14. Hammond P, Forster-Gibson C, Chudley AE, et al. Face-brain asymmetry in autism spectrum disorders. Mol Psych 13(6):614-623, 2008

15. Gor T, How Kau C, English jD, Lee RP, Borbely P. Three-dimensional comparison of facial morphology in white populations in Budapes, Hungary, and Houston, Texas. Am J Orthod Dentofacial Orthop 137:424-32, 2010

16. Hopman SMJ, Merks JHM, Suttie M, Hennekam RCM, Hammond P. Face shape differs in phylogenetically related populations. Eur J Hum Gen 22(1):1268-71, 2014

17. Kau CH, Richmond S, Zhurov A, et al. Use of 3-dimensionl surface acquisition to study facial morphology in 5 populations. Am J Orthod Dentofacial Orthop 137:S56.e1-S56.29, 2010

18. Claes $\mathrm{P}$, Walters $\mathrm{M}$, Shriver $\mathrm{MD}$ et al.: Sexual dimorphism in multiple aspects of 3D facial symmetry and asymmetry defined by spatially dense geometric morphometrics. J Anat 221(2):97-114, 2012

19. Asthana A, Lucey S, Goecke R. Regression based automatic face annotation for deformable model building. Pattern Recognition 44:2598-2613, 2011 
Table 1: Patient characteristics

\begin{tabular}{|c|c|c|c|c|}
\hline & & $\begin{array}{c}\text { GOSH: EBRT } \\
(\mathrm{N}=26)\end{array}$ & $\begin{array}{l}\text { EKZ-AMC: AMORE } \\
(\mathrm{N}=49)\end{array}$ & $P$ value \\
\hline Diagnosis age (yrs) & $\begin{array}{l}\text { Median (IQR) } \\
<10 \text { yrs }\end{array}$ & $\begin{array}{rr}5.1 & (2.2-6.3) \\
24 & \end{array}$ & $\begin{array}{rr}5.9 & (3.2-9.3) \\
39 & \end{array}$ & $0.25^{2}$ \\
\hline Attained age (yrs) & $\begin{array}{l}\text { Median (IQR) } \\
\geq 18 \mathrm{yrs}\end{array}$ & $\begin{aligned} 13.4 & (11.6-22.8) \\
10 & \end{aligned}$ & $\begin{aligned} 16.6 & (11.3-22.4) \\
22 & \end{aligned}$ & $0.85^{2}$ \\
\hline Follow-up (yrs) & Median (IQR) & $10.5 \quad(6.1-18.2)$ & $9.7 \quad(6.3-15.8)$ & $0.66^{2}$ \\
\hline Gender & $\begin{array}{l}\text { Male } \\
\text { Female }\end{array}$ & $\begin{aligned} 20 & (77 \%) \\
6 & (23 \%)\end{aligned}$ & $\begin{array}{ll}28 & (57 \%) \\
21 & (43 \%)\end{array}$ & $0.23^{1}$ \\
\hline Ethnicity & $\begin{array}{l}\text { European } \\
\text { Non-European }\end{array}$ & $\begin{array}{ll}15 & (58 \%) \\
11 & (42 \%)\end{array}$ & $\begin{aligned} 41 & (84 \%) \\
8 & (16 \%)\end{aligned}$ & $0.02^{1}$ \\
\hline Primary site & $\begin{array}{l}\text { PM } \\
\text { Orbit } \\
\text { HNNPM }\end{array}$ & $\begin{aligned} 15 & (58 \%) \\
8 & (31 \%) \\
3 & (12 \%)\end{aligned}$ & $\begin{array}{rr}23 & (47 \%) \\
20 & (41 \%) \\
6 & (12 \%)\end{array}$ & $0.71^{3}$ \\
\hline Side & $\begin{array}{l}\text { Left } \\
\text { Right } \\
\text { Midline }\end{array}$ & $\begin{aligned} 8 & (31 \%) \\
13 & (50 \%) \\
5 & (19 \%)\end{aligned}$ & $\begin{aligned} 21 & (43 \%) \\
21 & (43 \%) \\
7 & (14 \%)\end{aligned}$ & $0.58^{3}$ \\
\hline Nr of RTH Tx & $\begin{array}{l}0 \\
1 \\
2 \\
3\end{array}$ & $\begin{array}{r}1 \\
25\end{array}$ & $\begin{aligned} 4 & (8 \%) \\
35 & (71 \%) \\
8 & (16 \%) \\
2 & (4 \%)\end{aligned}$ & $0.05^{3}$ \\
\hline Initial local Tx & $\begin{array}{l}\text { No RTH } \\
\text { AMORE } \\
\text { EBRT } \\
\text { Proton }\end{array}$ & $\begin{array}{cl}1 & (4 \%) \\
25 & (96 \%)\end{array}$ & $\begin{aligned} 12 & (24 \%) \\
25 & 51 \%) \\
10 & (20 \%) \\
2 & (4 \%)\end{aligned}$ & $0.02^{3}$ \\
\hline
\end{tabular}

GOSH; Great Ormond Street Hospital, EKZ-AMC; Emma Children's Hospital-Academic Medical Centre, Yrs; years, IQR; interquartile range, PM; parameningeal, HNNPM; head and neck non parameningeal, Tx; treatment, RTH; radiotherapy, AMORE; Ablative surgery, MOulage brachytherapy and REconstruction, EBRT; external beam radiotherapy

${ }^{1}$ Chi square

${ }^{2}$ Mann Whitney U

${ }^{3}$ Fisher exact 
Table 2: Treatment description and tumour localization of survivors with asymmetry index $>5$.

\begin{tabular}{|c|c|c|c|c|c|}
\hline$\overline{A S I}$ & $\begin{array}{l}\text { Age at } \\
\text { diagnosis }\end{array}$ & $\begin{array}{l}\text { Age at } \\
\text { scan }\end{array}$ & Ethnicity & Treatment & Specifications \\
\hline \multicolumn{6}{|l|}{ EBRT } \\
\hline 6.2 & 2.5 & 9.7 & Caucasian & EBRT, $S$ & Orbit \\
\hline 6.5 & 9.1 & 13.5 & Caucasian & EBRT & Retropharyngeal, infratemporal region \\
\hline 7.5 & 5.2 & 20.0 & Caucasian & EBRT & Orbit \\
\hline 8.9 & 6.2 & 26.5 & Caucasian & EBRT & Middle ear, extension middle fossa \\
\hline 9.5 & 1.3 & 12.3 & Non-European & EBRT & $\begin{array}{l}\text { Right petrous apex and body sphenoid, } \\
\text { ICE (cavernous sinus), ECA. Separate } \\
\text { lymph node mass. }\end{array}$ \\
\hline 10.8 & 6.3 & 16.5 & Non-European & EBRT & Orbit \\
\hline 11.7 & 7.9 & 26.5 & Non-European & EBRT & Middle ear, petrous bone \\
\hline 14.4 & 6.0 & 27.9 & Non-European & EBRT & Left orbit and posterior nasal space \\
\hline 14.9 & 1.0 & 18.7 & Non-European & $\mathrm{S}$ & Temporal \\
\hline \multicolumn{6}{|l|}{ AMORE } \\
\hline 5.6 & 3.0 & 19.7 & Caucasian & S, AMORE ${ }^{1}$ & Submandibular glands \\
\hline 6 & 13.4 & 19.6 & Caucasian & AMORE & $\begin{array}{l}\text { Masticator space, maxillary sinus, cheek, } \\
\text { left palate }\end{array}$ \\
\hline 7.5 & 4.2 & 13.7 & Caucasian & $\begin{array}{l}\text { AMORE, } \\
\text { EBRT }^{1}\end{array}$ & Infratemporal fossa, orbit, maxillary wall \\
\hline 7.5 & 7.5 & 11.5 & Caucasian & AMORE & Right orbit and frontal bone \\
\hline 8.2 & 7.4 & 15.5 & Caucasian & $\mathrm{AMORE}^{2}$ & Ala nasi, extension to palate \\
\hline 8.5 & 1.7 & 5.7 & Caucasian & AMORE & Temporal region \\
\hline 19.1 & 9.9 & 13.7 & Non-European & AMORE & $\begin{array}{l}\text { Left middle ear, mastoid, external } \\
\text { meatus }\end{array}$ \\
\hline 10.6 & 7.7 & 14.9 & Caucasian & AMORE & Orbit \\
\hline 10.8 & 10.2 & 19.8 & Caucasian & $\begin{array}{l}\text { AMORE, } \\
\text { EBRT }^{1}\end{array}$ & $\begin{array}{l}\text { Infratemporal fossa, maxillary sinus, } \\
\text { parapharyngeal, ICE }\end{array}$ \\
\hline 11.0 & 5.3 & 16.8 & Caucasian & AMORE & Parotid gland \\
\hline 14.1 & 10.5 & 26.6 & Caucasian & EBRT & $\begin{array}{l}\text { Cheek, locoregional lymp nodes } \\
\text { (submandibular) }\end{array}$ \\
\hline 14.6 & 1.1 & 9.8 & Caucasian & $\begin{array}{l}\text { EBRT, } \\
\text { AMORE }^{1,3}\end{array}$ & Orbit \\
\hline 15.3 & 11.0 & 30.1 & Caucasian & $\begin{array}{l}\text { S, AMORE } \\
\text { EBRT }^{1} \\
\text { AMORE }^{1,3}\end{array}$ & Orbit \\
\hline 16.5 & 2.0 & 15.3 & Caucasian & AMORE & Pterygoid loge, parotid gland \\
\hline 17.9 & 4.8 & 9.8 & Caucasian & $\begin{array}{l}\text { EBRT, } \\
\text { AMORE }^{1,3}\end{array}$ & Orbit \\
\hline
\end{tabular}

ASI; asymmetry index (adjusted for age), EBRT; external beam radiotherapy treatment

group, AMORE; Ablative surgery, MOulage brachytherapy and REconstruction, ICE;

intracranial extension, age at scan; age at evaluation, S; surgery

${ }^{1}$ Salvage treatment 
${ }^{2}$ Reconstruction failed initially

${ }^{3}$ Exenterated orbit 
Figure 1: Asymmetry index plotted against age and specified for treatment group and ethnic background

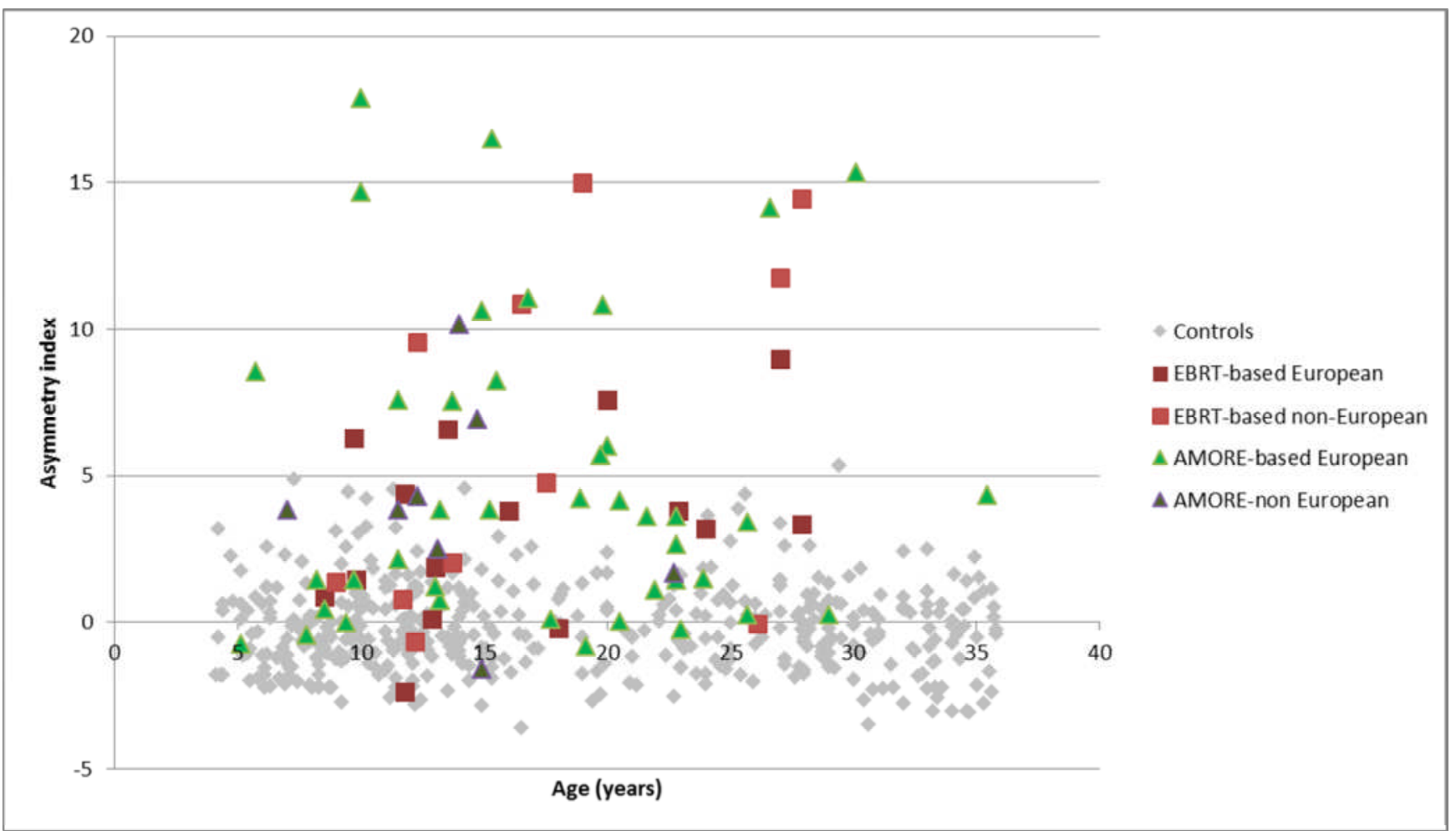


Figure 2: normalized facial asymmetry with respect to race, sex and age at each of 25,000 points on the face surface and at 2SD compared to matched mean (powerpoint) 
Figure 3: histogram representing signature weights for the Dutch and British survivors and controls

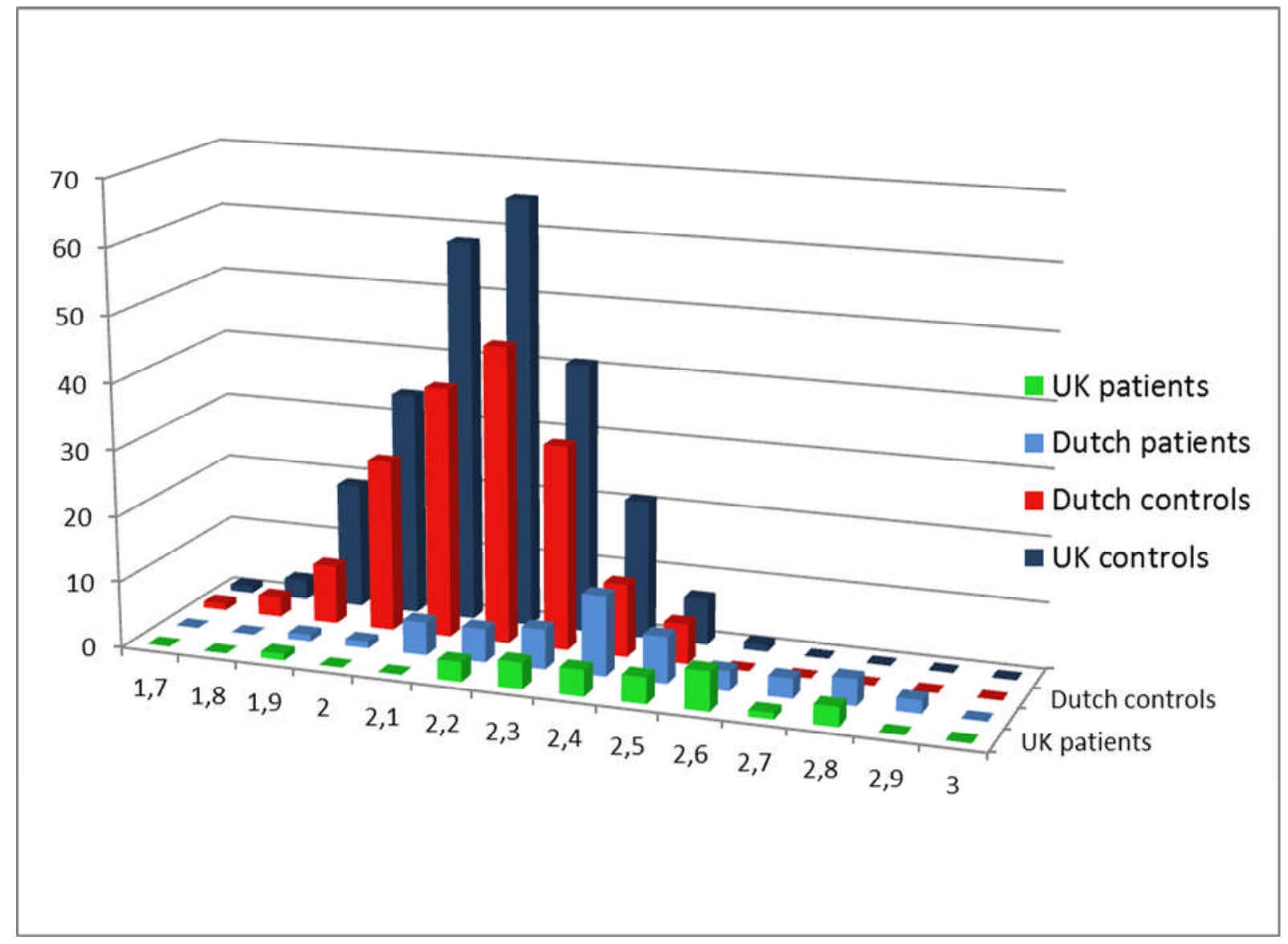


Figure 4: clinical assessment of asymmetry related to the raw asymmetry index, based on 3D

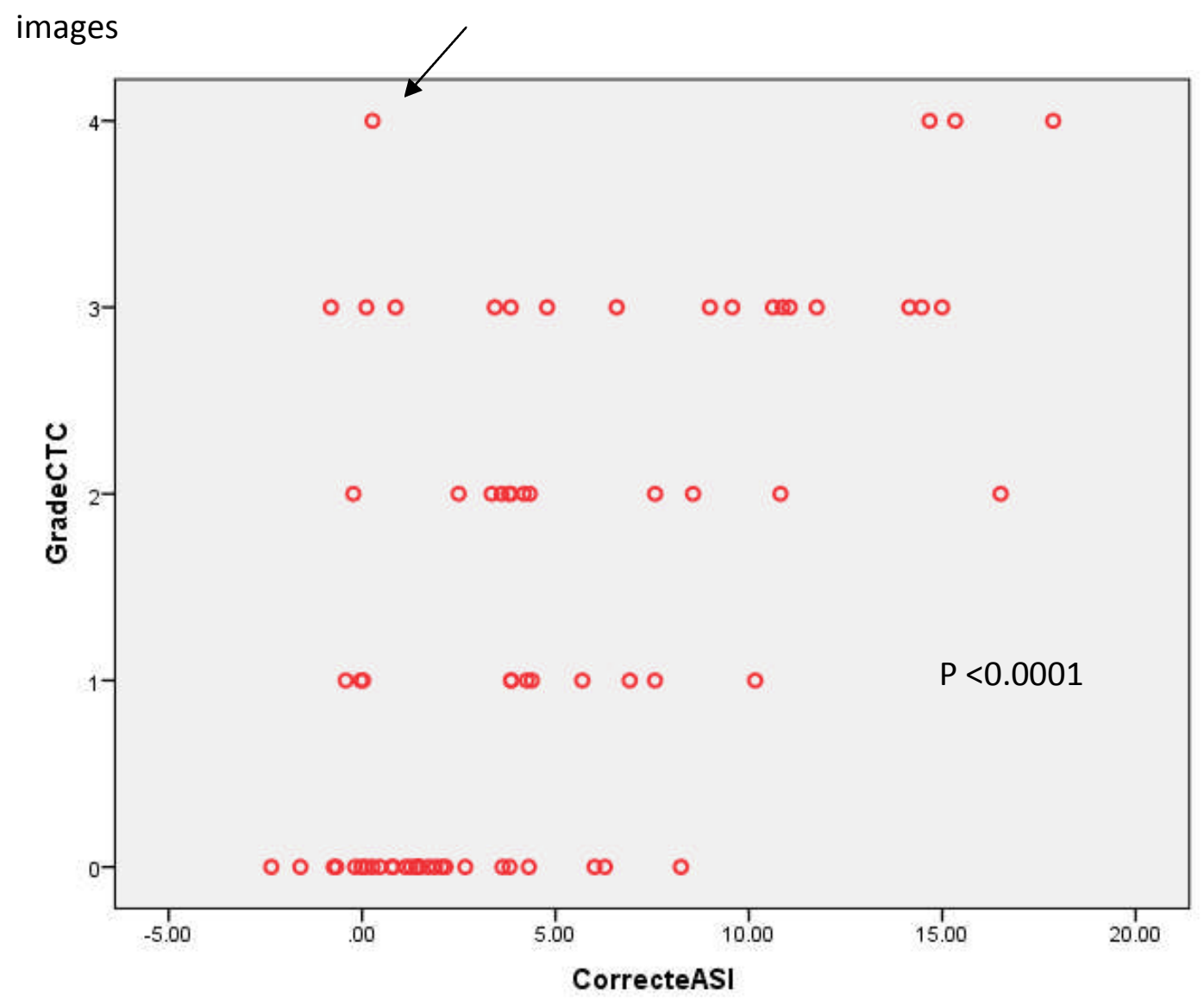

Arrow is directed at survivor with exenterated orbit, wearing prosthesis during 3D image capture.

Appendix 1: landmarks (will follow) 